\title{
Fatores pessoais e sociais que influenciam o bem-estar subjetivo: diferenças ligadas estatuto socioeconômico
}

\author{
Personal and social factors that influence subjective well-being: \\ socioeconomic differences
}

Tania Gaspar ${ }^{1}$

Leonor Balancho ${ }^{1}$
${ }^{1}$ Universidade Lusíada de Lisboa. R. Junqueira 188-198. 1349-001 Lisboa Portugal. tania.gaspar. barra@gmail.com

\begin{abstract}
The main objective is to study the relation and impact of Socioeconomic Status (SES) on the subjective well-being in children and adolescents and the role of social and personal factors in this relation. Data was collected in 16 schools; 8 in the North and 8 in the Lisbon Region of Portugal. The questionnaires were filled out by 1,181 youths, $51.5 \%$ of which were female. Ages ranged between 8 and 17, the girl's average age being 9.8 years $(S D=1.32)$ and the boy's average age being 10.0 (SD = 1.53); $2.6 \%$ of young people had special educational needs, and $3 \%$ did not use the Portuguese language at home; $12.2 \%$ had repeated a grade; $27.1 \%$ had a high SES; $64.2 \%$ had medium/low SES and $8.7 \%$ were unemployed. Three adequate regression models were built. Model 1 established the association between SES and subjective well-being. This association turned out to be not significant with the effect of personal and social factors, which were strongly associated with subjective wellbeing. It was revealed that the impact of SES on subjective well-being is mitigated by the presence of social and personal factors. The promotion of personal and social skills appears to be an effective way to avoid the negative effects of low SES in child and adolescent development.

Key words Socioeconomic status, Subjective well-being, Psychosocial factors, Children and adolescents
\end{abstract}

Resumo Pretende-se compreender e caracterizar a associação entre o Estatuto Socioeconômico (ESE) e o bem-estar subjetivo em crianças e adolescentes e ainda entender a influência que fatores pessoais e sociais podem ter nela. Os dados foram recolhidos em 16 escolas, uma metade no Norte e outra na Região de Lisboa, Portugal. A amostra envolve 1.181 estudantes, $51,5 \%$ do gênero feminino, cuja idade varia entre 8 e 17 anos, com média de 9,9 anos $(D P=1,42)$. Dos alunos, 2,6\% têm necessidades educativas especiais, enquanto que 3\% não utilizam o idioma português em casa. Foram retidos por pelo menos um ano 12, 2\%. Quanto ao ESE, $27,1 \%$ o têm alto, $64,2 \%$ médio/baixo, enquanto que $8,7 \%$ estão desempregados. Foram construídos três modelos de regressão adequados. O Modelo 1 estabelece uma associação entre a ESE e o bem-estar subjetivo. Esta associação tornou-se não significativa com o efeito de fatores pessoais e sociais, que foram fortemente associados com o bem-estar subjetivo. Verifica-se que o impacto do ESE no bem-estar subjetivo é atenuado pela presença de fatores pessoais e sociais. A promoção de competências pessoais e sociais parece ser uma forma de prevenção dos efeitos negativos do ESE baixo no desenvolvimento das crianças e adolescentes. Palavras-chave Estatuto socioeconômico, Bem -estar subjetivo, Crianças adolescentes 


\section{Introdução}

O impacto do Estatuto Socioeconômico (ESE) nos níveis de bem-estar da população em geral, bem como de grupos etários específicos, como as crianças e adolescentes, ou de grupos sociais particulares, como a família, tem sido bem documentado pelas ciências sociais e humanas. Os dados empíricos e os modelos teóricos têm emergido de domínios como a psicologia, a sociologia, a economia e a política social, enquanto que os avanços recentes nos estudos nas áreas da qualidade de vida e do bem-estar têm ajudado a promover uma compreensão alargada, mas ainda não suficiente, da relação entre estes dois fenômenos $^{1-5}$.

O conceito de bem-estar tem surgido com diferentes entendimentos. Alguns autores estudam o subjetivo ${ }^{2}$, outros o psicológico ${ }^{6,7}$, e os demais o físico, através da perceção pessoal sobre a saúde. A integração destas três perspetivas é essencial para se entender a complexidade dos processos que lhe estão inerentes, e é também capital para esclarecer alguns dados empíricos, por vezes contraditórios, que vão emergindo nesta área de confluência.

O bem-estar subjetivo inclui 3 elementos: o cognitivo, ou de satisfação global com a vida; os afetos positivos; e os afetos negativos ${ }^{3}$. Por seu lado, o bem-estar psicológico é, de acordo com Ryff $^{6}$ e Ryff e Keyes ${ }^{7}$, um composto de seis elementos, que inclui: Autonomia; Controle sobre o ambiente; Crescimento Pessoal; Relações positivas com os outros; Propósito na vida; e, Autoaceitação.

Uma investigação indica que o ESE é um fator crítico que influência a qualidade de vida e o bem-estar de crianças, adolescentes e famílias ${ }^{8}$. O ESE influencia o funcionamento humano de múltiplas formas, em particular nos processos de desenvolvimento ao longo do ciclo de vida e na saúde psicológica e física. Por isso, hoje é inquestionável considerar que a pobreza seja fator de risco para a saúde e o bem-estar do indivíduo ao nível físico, mental e social'.

O baixo ESE e os seus correlatos, como o menor nível educacional, a pobreza e a fraca saúde, afetam a sociedade como um todo, tendo efeitos nefastos, em particular, nas crianças. As crianças e os adolescentes com estatuto socioeconômico desfavorecido apresentam um conjunto mais vasto de riscos, pois estão mais frequentemente expostos a múltiplos stresses, oriundos de numerosas origens, como maior probabilidade de doenças físicas, tensões psicológicas e relacionais a nível familiar e menor apoio social ${ }^{10}$. O ESE da família de origem, e as fragilidades e exclusões que lhe estão associadas, são preditores relevantes da qualidade de vida das gerações mais novas, tendo impacto no seu desenvolvimento socioemocional, cognitivo e físico. O fato de a família ser numerosa, pertencente a uma minoria étnica, viver em zonas habitacionais de carência, e os pais estarem desempregados e terem baixos níveis educacionais, está claramente associado a uma baixa perceção de bem-estar no domínio físico, e a uma baixa perceção da qualidade de vida, na perspetiva das crianças e dos adolescentes dessas famílias, em especial na relação com a saúde.

As desigualdades na distribuição da riqueza e de recursos, e na qualidade de vida, estão a aumentar em muitos países desenvolvidos. A sociedade se beneficiará de um aumento de foco nas fundações e causas destas desigualdades, e de maiores esforços para reduzir a distância entre os vários níveis socioeconômicos, e aliviar as disparidades, quer ao nível individual, quer social. De acordo com dados publicados pela American Psychologycal Association', o impacto do ESE nas populações mais novas e nas áreas de saúde psicológica, física, educacional e familiar, é o seguinte: a) Na Saúde Psicológica - Há crescente evidência que suporta a ligação entre baixo ESE e resultados negativos na saúde psicológica ${ }^{11}$. Níveis mais elevados de dificuldades emocionais e comportamentais, incluindo ansiedade, depressão, deficit de atenção/hiperatividade e transtornos de comportamento ${ }^{12}$; Níveis mais altos de agressividade ${ }^{13}$, hostilidade, perceção de ameaça e perceção de discriminação nos grupos de adolescentes ${ }^{14}$, Maior incidência da doença de Alzheimer mais tarde na vida ${ }^{15-18}$; b) Saúde Física - encontra-se uma relação entre o ESE e a saúde, quer no momento do nascimento, quer durante toda a vida. Assim, menores níveis de ESE foram encontrados associados com o seguinte: Maior probabilidade de ser sedentário ${ }^{14}$, e maior índice de massa corporal em adolescentes ${ }^{17}$. A razão pode ser a falta de recursos no bairro onde habitam, como parques infantis, e a falta de opções acessíveis para comer alimentos saudáveis; Marcadores fisiológicos mais elevados associados a experiências estressantes crônicas em adolescentes ${ }^{17}$; Taxas mais elevadas de doença cardiovascular em adultos ${ }^{19-21}$; c) Educação - Encontra-se uma relação entre ESE e resultados e envolvimento escolares: O nível socioeconômico parece criar lacunas de desempenho associados a certas minorias étnicas, como é o caso das crianças negras e hispânicas, quando comparadas com os 
níveis de escolaridade das caucasianas ${ }^{22}$; Crianças de famílias de baixo ESE começam, muitas vezes, o jardim-de-infância com muito menos conhecimentos linguísticos ${ }^{23}$; Crianças de lares desfavorecidos têm pelo menos $10 \%$ de valores inferiores à média nacional na pontuação em matemática e leitura ${ }^{24}$; Crianças que vivem em ambientes pobres são muito mais propensas a se ausentar da escola no decorrer das suas experiências educacionais $^{25}$, aumentando ainda mais a diferença de aprendizagem entre eles e os seus pares mais $\operatorname{ricos}^{26}$; d) Bem-estar familiar - Evidências empíricas indicam que a situação socioeconômica afeta a estabilidade da família, incluindo as práticas parentais e os consequentes resultados no desenvolvimento das crianças ${ }^{27}$. A pobreza é também um indicador fiável de abuso infantil e negligência. Entre as famílias de baixo rendimento, os que são expostos a pais com problemas de abuso de substâncias apresentam as maiores taxas de abuso infantil e de negligência ${ }^{28}$, Menor ESE tem sido associado ao adensamento doméstico, ou excesso de elementos a viverem em conjunto, uma condição que tem consequências negativas para adultos e crianças, incluindo maior stresse psicológico e resultados de saúde mais fracos ${ }^{29}$. O investimento na avaliação subjetiva do bem -estar, na perspetiva das crianças, foi alvo de um estudo recente da $\mathrm{UNICEF}^{30}$ que relembra que estas são o recurso mais importante de qualquer país, e que, quer do ponto de vista econômico, quer moral, investir na saúde, na educação e nas aptidões das crianças traz os mais elevados retornos a nível nacional e mundial.

Pretende-se compreender e caracterizar a associação entre o ESE e o bem-estar subjetivo em crianças e adolescentes. Temos também o objetivo de compreender a influência, ou o efeito, que fatores pessoais (otimismo, autoestima e resiliência) e sociais (satisfação com suporte social de amigos e família) podem ter nessa associação entre ESE e o bem-estar subjetivo.

\section{Metodologia}

\section{Amostra}

Com o objetivo inicial de recolher 1200 questionários, 600 de cada uma das Zonas (Norte e Sul do País) dos quais, 300 participantes do $1^{\circ}$ ciclo e 300 do $2^{\circ}$ em cada uma delas. Foi uma amostra por conveniência dentro dos critérios da amostragem. Deste modo, os critérios de inclusão no estudo foram: (a) ao nível das escolas a zona do
País (Zona Norte e Sul do País) e a disponibilidade da Direção das escolas para participar; (b) ao nível dos alunos foram incluídos todos aqueles das escolas que aceitaram participar no estudo, que obtiveram o consentimento dos encarregados de educação, que aceitaram participar voluntariamente e com capacidade cognitiva para fazer o autopreenchimento dos questionários. Foram excluídos do estudo as escolas que não aceitaram participar, e os alunos que não receberam o consentimento do encarregado de educação, ou que não aceitaram participar voluntariamente ou sem capacidade cognitiva para preencher sozinho os questionários.

Os dados foram recolhidos em 16 escolas, 8 na Região Norte (RN) e 8 na Região de Lisboa (RL), Portugal. Quanto às crianças e adolescentes, 1181 preencheram os questionários $(33.4 \%$ na $\mathrm{RN}$ e $66.6 \%$ na $\mathrm{RL}), 51.5 \%$ são raparigas com uma média de idades de 9.8, variando entre 8 e 17 anos, e os rapazes com uma média de idade de 10.0 ( $\mathrm{SD}=1.53)$. Apenas $2.6 \%$ dos jovens têm necessidades educativas especiais, e 3\% não usam a língua Portuguesa em casa. Já tinham reprovado um ano, $12.2 \%$. Têm alto ESE, $27,1 \%$ e médio/baixo, 64,2\%, enquanto $8,7 \%$ estão desempregados. O ESE foi avaliado tendo como base a escala de Grafar. No questionário foi perguntado aos alunos qual a profissão do pai e sua escolaridade. Estes dados foram categorizados pelos investigadores num valor de 1 a 7 ( 1 nível socioeconômico muito elevado -5 nível socioeconômico muito baixo, 6 reformado e 7 desempregado).

\section{Instrumentos}

Com o objetivo de medir o bem-estar subjetivo e os fatores pessoais e sociais associados, tevese como base o modelo apresentado por Matos et al. ${ }^{31}$ e foram utilizados os seguintes instrumentos para as medir:

\section{Bem-estar subjetivo}

Os itens utilizados para avaliar esta variável foram retirados da versão completa da escala KIDSCREEN-10. A boa consistência interna de fidelidade (Cronback's alpha $=0,82$ ) e a boa fidelidade/estabilidade teste-reteste $(r=0.73$; ICC $=$ 0.72) permitem avaliar de forma precisa e estável a HRQoL.

A escala KIDSCREEN-10 permite diferenciar grupos; resultados baixos referem-se a sentimentos de tristeza, desajustados e insatisfeitos relativamente à vida familiar, pares e vida escolar, 
e resultados altos indicam o oposto: sentimentos de felicidade, ajustados e satisfeitos com família, escola e grupo de pares.

O instrumento resulta num valor global, onde uma medida unidimensional representa o valor global das versões completas do KIDSCREEN (KIDSCREEN-52 e KIDSCREEN-27), adequado para estudos grandes e epidemiológicos (The KIDSCREEN Group Europe, 2006). De acordo com as orientações internacionais, a tradução do questionário KIDSCREEN incluiu um processo de Backtranslation. A versão portuguesa apresenta boas propriedades métricas e a análise fatorial confirmatória tem um modelo ajustado ${ }^{31}$.

\section{Resiliência}

A escala de resiliência foi elaborada com base no International Resilience Research Project ${ }^{32}$. O método de retradução foi utilizado para traduzir e adaptar a escala. A escala foi traduzida por dois investigadores portugueses, e as duas versões foram comparadas e integradas. A versão resultante deste processo foi retraduzida para Português e revista por um investigador bilingue, e comparada com a versão original. A versão portuguesa final foi testada com a sua aplicação a algumas crianças e adolescentes para adaptar a língua. Depois deste processo, a versão portuguesa foi obtida. A escala de Grotber integra três dimensões correlacionadas de resiliência, organizadas consoante suas fontes: suporte externo obtido, desenvolvimento de forças internas e competências interpessoais e de resoluções de problemas adquiridas. Estas dimensões foram identificadas, respetivamente, como Eu Tenho, Eu Sou e Eu Consigo $^{32}$, utilizadas para lidar com adversidades. Como a versão original, foram utilizados cinco itens (variáveis manifestas) para cada dimensão. A versão portuguesa apresenta fidelidade, validade convergente e discriminativa. A escala foi revalidada e os resultados apresentam invariância dos parâmetros ${ }^{33}$.

\section{Otimismo}

O Life Orientation - Revised Test ${ }^{34}$ é um instrumento de autopreenchimento composto por dez itens, quatro dos quais são distratores e seis avaliam o otimismo. As respostas possíveis são apresentadas numa escala ordinal de cinco pontos, variando entre "Concordo Totalmente" e "Discordo Totalmente". Os sujeitos registam a afirmação com a qual concordam em relação a cada um dos itens apresentados. O Life Orien- ted - Revised Test ${ }^{34}$ foi traduzido e adaptado para crianças e adolescentes portugueses ${ }^{10}$ com uma consistência interna de $\alpha=.59$.

\section{Autoestima}

A variável autoestima foi avaliada com a versão Portuguesa da Kelley's scale Hare Self-Esteem Scale ${ }^{35}$, traduzida e adaptada através do processo de retradução, o mesmo utilizado e apresentado acima para a variável resiliência. As propriedades métricas da versão portuguesa do instrumento estão a ser preparadas ${ }^{36}$.

Esta escala tem 18 itens num formato Likert, variando entre "Concordo Totalmente" e "Discordo Totalmente", com três dimensões da autoestima: relacionada com família, com amigos e com a escola.

\section{Satisfação com Suporte Social}

A escala de Satisfação com o Suporte Social ${ }^{37}$ foi construída para populações de jovens adultos e de adultos, em situações de doença, tanto crônica como psicológica. Para a construção desta escala, um grupo de dimensões relacionadas com saúde e bem-estar foi considerada, paralelamente a outras. A versão original da escala é composta por 15 frases afirmativas para autopreenchimento. Os sujeitos marcam o nível com o qual concordam com a afirmação (caso se aplique ao indivíduo), numa escala tipo Likert, variando entre "Concordo Totalmente" e "Discordo Totalmente". Os 15 itens estão distribuídos entre quatro dimensões ou fatores, gerados empiricamente, para medir os seguintes aspetos relacionados com a Satisfação com o Suporte Social: "Satisfação com Amizades", "Intimidade", "Satisfação com a Família" e "Atividade Social". A Escala de Satisfação com o Suporte Social ${ }^{31}$ foi traduzida e adaptada para crianças e adolescentes portugueses ${ }^{38} \mathrm{e}$ obteve uma consistência interna de $\alpha=.77$.

\section{Recolha de dados e análise}

Este estudo faz parte de uma investigação mais alargada que tem o objetivo de avaliar as variáveis associadas ao bem-estar em crianças e adolescentes portugueses. As escolas de ambas as zonas (Norte e Sul) foram contactadas. Com as que aceitaram participar do estudo foi acordado o procedimento. Numa primeira fase foram entregues as cartas de consentimento informado para os pais, depois de assinadas foi combinado um dia para o investigador fazer a recolha 
em contexto de sala de aula com a presença de um professor. Todos os questionários foram autopreenchidos em português. Foram asseguradas as questões éticas do estudo e obtido o consentimento dos alunos e dos pais para a participação. A confidencialidade e a anonimidade foram preservadas. A recolha de dados teve lugar entre fevereiro e maio de 2012.

Para a análise de dados foi utilizado o software SPSS 20, e para a estatística descritiva, a ANOVA e as regressões lineares.

\section{Resultados}

Foram encontradas diferenças estatisticamente significativas entre os alunos com ESE médio/ baixo e os com elevado. Os alunos com ESE elevado têm melhores indicadores de saúde quando comparados aos com médio/baixo. Os alunos com ESE elevado apresentam valores maiores em relação ao bem-estar subjetivo, ao otimismo, à autoestima e à satisfação com o suporte social. Não foram encontradas diferenças estatisticamente significativas em relação à resiliência em ambos os grupos de ESE (Tabela 1).

Foram desenvolvidos três modelos de regressão linear. $\mathrm{O}$ modelo 1 tem o objetivo de estudar o impacto do ESE no bem-estar subjetivo das crianças e adolescentes; no modelo 2, além de incluir o SES, também se pretende observar o impacto de características pessoais (otimismo, autoestima e resiliência) no bem-estar subjetivo; no modelo 3, além do ESE e das características pessoais, foi introduzida a variável social da satisfação com o suporte social (amigos e família).

Em relação ao Modelo de Regressão 1, foi encontrado um adequado [ $\mathrm{F}(1,392) 4,45$; p <,036] que justifica $1 \%$ da variância explicada. O ESE tem uma associação estatisticamente significativa com o bem-estar subjetivo. Em relação ao modelo de regressão 2 , foi alcançado um adequado [F $(4,389) 58,06, \mathrm{p}<0,001]$, com a variância explicada de $36,7 \%$. Todas as características pessoais (otimismo, autoestima e resiliência) estão associadas ao bem-estar subjetivo, enquanto que a variável ESE deixa de ter um impacto estaticamente significativo nele. Em relação ao modelo de regressão 3 , foi alcançado um adequado [F $(5,388) 55,03, \mathrm{p}<0,001]$, com a variância explicada de 40,7\%. A variável satisfação com o suporte social e todas as características pessoais (otimismo, autoestima e resiliência) estão fortemente associadas ao bem-estar subjetivo. O ESE continua sem ter um impacto estaticamente significativo no bem-estar subjetivo (Tabela 2 ).

\section{Discussão}

O principal objetivo é compreender e caracterizar a associação entre o bem-estar subjetivo e o ESE em crianças e adolescentes, bem como o impacto de características sociais e pessoais nesta relação. Os dados revelaram diferenças estatísticas significativas entre as crianças e os adolescentes com ESE baixo e com elevado. Os estudantes com ESE elevado apresentam indicadores sociais e de saúde pessoal mais positivos, são mais otimistas, apresentam autoestima mais elevada e maior satisfação com o suporte social, relacionadas com os pais e os amigos. Bem-estar subjetivo e qualidade de vida subjetiva envolvem o apoio social e o ajustamento psicossocial, assim como as competências relacionadas com autoestima, resiliência, otimismo, stress e coping ${ }^{35,39-41}$. Crianças com mais fatores de risco e menos de proteção apresentam bem-estar subjetivo mais negativo. Verifica-se uma forte associação entre o bem-estar subjetivo e o ESE. O ESE elevado está positivamente

Tabela 1. ANOVA - análise das diferenças de ESE ligadas a caraterísticas sociais e pessoais de crianças e adolescentes.

\begin{tabular}{|c|c|c|c|c|c|c|c|}
\hline \multicolumn{8}{|c|}{ Estatuto Socioeconômico } \\
\hline \multirow[t]{2}{*}{ Dimensões } & \multicolumn{2}{|c|}{ ESE Elevado } & \multicolumn{2}{|c|}{ ESE Médio/baixo } & \multicolumn{2}{|c|}{ Desempregado } & \multirow[t]{2}{*}{$\mathbf{F}$} \\
\hline & M & DP & $\mathbf{M}$ & DP & M & DP & \\
\hline Kidscreen 10 & 43.64 & 5.02 & 42.05 & 5.19 & 41.78 & 4.98 & $5.64^{* *}$ \\
\hline Otimismo & 23.29 & 3.73 & 22.16 & 3.68 & 21.67 & 3.63 & $6.50^{\star *}$ \\
\hline Resiliência & 65,63 & 8,03 & 64,27 & 9,37 & 63,28 & 10,07 & (n.s.) \\
\hline Autoestima & 59.09 & 7.48 & 56.63 & 8.21 & 59.00 & 7.63 & $5.01^{\star *}$ \\
\hline Satisfação Suporte Social & 46.64 & 6.88 & 45.27 & 7.26 & 43.75 & 6.19 & $4.13^{\star *}$ \\
\hline
\end{tabular}

$* \star \mathrm{P}<, 01$ 


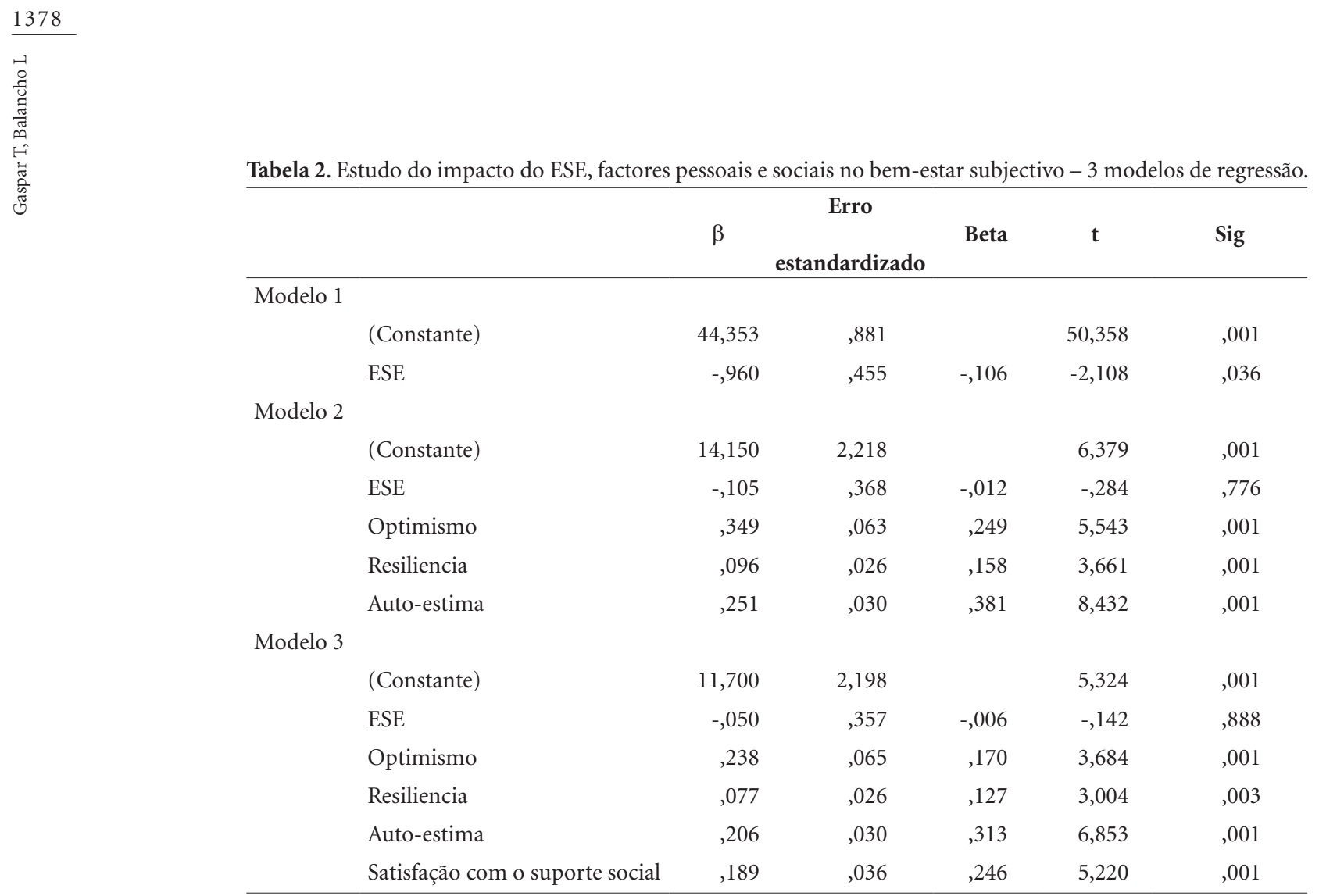

Variável Dependente - bem-estar subjectivo.

relacionado ao bem-estar subjetivo ${ }^{35}$. O ESE tem inequívocas consequências nos indivíduos, nos grupos familiares, nas comunidades e nas nações. Essas consequências são gravosas a muitos níveis, sendo um deles a perceção subjetiva e psicológica de bem-estar. Objetivamente, a gravidade do impacto do baixo ESE revê-se em áreas como a saúde física e psicológica, a qualidade dos cuidados parentais, sociais e das relações educativas, assim como nos impactos desenvolvimentistas diversos. Desse modo, desemprego, pobreza, exclusão e marginalidade de algum tipo, tantas vezes associados ao baixo ESE, são fatores de grave risco social e psicológico ${ }^{13}$. Se a riqueza tem mostrado estar associada a vários tipos de sucessos, e a bons resultados na vida, a pobreza - entendida em sentido lato, mas sobretudo delimitada por um baixo ESE - implica um maior risco de infelicidade e a um maior mal-estar, dado o menor suporte social, as relações familiares e parentais mais fragilizadas e, por vezes, mais violentas, comportamentos na área da saúde promotores de mais vulnerabilidade, menos acesso a recursos como boa e estável educação escolar e ambientes físicos harmoniosos, menor autoaceitação e mais fraco sentido de controle sobre a vida ${ }^{8,28,32,42}$.

A fim de entender o impacto das características pessoais, sociais e do ESE no bem-estar subjetivo dos alunos foram introduzidas outras variáveis pessoais, o otimismo, a resiliência e a autoestima, e sociais: satisfação com o suporte social. O modelo final, com todas as variáveis, permite concluir que as características pessoais e sociais apresentam um valor explicativo mais forte no bem-estar subjetivo em crianças e adolescentes, e na presença desses fatores, a associação entre o ESE e o bem-estar torna-se não estatisticamente significativa. Assim, fatores pessoais e sociais podem ser considerados como de proteção, se relacionado o impacto do ESE no bem -estar subjetivo. A prevenção do insucesso escolar e da intervenção deve incluir o aspecto social e a promoção de competências pessoais, envolvendo estudantes, professores amigos, família e comunidade $^{34}$. A promoção da qualidade de vida em crianças e adolescentes requer uma abordagem ecológica ${ }^{43,44}$ e de desenvolvimento.

Num momento gravoso para as sociedades desenvolvidas, em que a crise e a austeridade têm diminuído o uso dos recursos adequados pelas crianças, limitado seu acesso a serviços de educação, saúde e bem-estar, e restringido as oportunidades delas e dos jovens de participar ativa e plenamente na vida familiar e social. Pois, como nos diz um recente relatório da Eurochild ${ }^{45}$, as consequências a longo prazo da fragilidade econômica, 
do desemprego, e da sua recente subida exponencial, ou das condições de emprego precárias, podem ser sérias e dificilmente recuperáveis, tanto a nível do bem-estar das crianças, como dos pais. A pobreza infantil e a que afeta as gerações mais novas é o flagelo mais dramático das sociedades atuais e, podendo e precisando ser evitada, deveria implicar em praticas ativas de proteção dos mais vulneráveis e de retorno a oportunidades e a apoios prestados a crianças e adolescentes.

O estudo tem algumas limitações, como se trata de amostra Nacional, não se pode generalizar de modo linear para outras populações. Propõe-se o estudo destas variáveis em outras populações e nações.

\section{Colaboradores}

T Gaspar fez a descrição metodológica, a análise e a discussão dos dados, e L Balancho a revisão de literatura.
No entanto, considera-se que o presente estudo é um importante contributo para a compreensão do impacto do ESE no bem-estar de crianças e adolescentes.

O desenvolvimento de competências pessoais e sociais pode funcionar como proteção ao impacto negativo de níveis mais baixos de estatuto socioeconômico ou situação de desemprego parental. Em termos de intervenção ao nível da promoção de saúde, é fundamental intervir junto aos grupos mais desfavorecidos e em situação de maior risco de um desenvolvimento pouco saudável. A um nível macro, reduzindo os níveis de pobreza, e a um nível meso, diminuindo seus riscos, quando presente.

\section{Referências}

1. Diener E, Larsen RJ. Temporal stability and cross-situational consistency of affective, behavioral and cognitive responses. J Pers Soc Psychol 1984; 47(4):580-592.

2. Diener E, Sandvik E, Seidlitz L, Diener M. The relationship between income and subjective well-being: Relative or absolute? Social Indicators Research 1993: 28(3):195-223.

3. Diener E, Biswas-Diener R. Will money increase subjective well-being? A literature review and guide to needed research. Social Indicators Research 2002; 57(2):119-169.

4. Diener E, Oishi S. Money and happiness: Income and subjective well-being across nations. In: Diener E, Suh EM, editors. Culture and subjective well_being. Cambridge: MIT Press; 2000. p. 185-218

5. Diener E, Diener C. The wealth of nations revisited: Income and quality of life. Social Indicators Research 1995; 36(3):275-286.

6. Ryff C. Happiness is everything, or is it? Explorations on the meaning of psychological well-being. J Pers Soc Psychol 1989; 57(6):1069-1081.

7. Ryff C, Keyes C. The structure of psychological well-being revisited. J Pers Soc Psychol 1995; 69(4):719-727.

8. Gaspar T. Health-Related quality of life in children and adolescents: Personal and social fators that promote quality of life. German: Lambert Academic Publishing; 2010.

9. Gaspar T, Matos MG, Ribeiro JL, Leal I. Qualidade de Vida em Crianças e Adolescentes: Impacto da Migração e Estatuto Socio-Econômico. Brazilian Journal of Health $2010 ; 1(1): 80-92$

10. Gaspar T. Promoção do bem-estar na adolescência: Impacto do estatuto socio-econômico e do estatuto migrante. Aná. Psicológica 2008; 26(2):265-279.

11. Newacheck PW, Hung YY, Park MJ, Brindis CD, Irwin CE. Disparities in adolescent health and health care: Does socioeconomic status matter? Health Serv Res 2003; 38(5):1235-1252. 
12. Weissman MM, Leckman JF, Merikangas KR, Gammon GD, Prusoff BA. Depression and anxiety disorders in parents and children: Results from the Yale Family Study. Arch Gen Psychiatry 1984; 41(9):845-852.

13. Molnar BE, Cerda M, Roberts AL, Buka SL. Effects of neighborhood resources on aggressive and delinquent behaviors among urban youths. Am J Public Health 2008; 98(6):1086-1093.

14. Chen E, Paterson LQ. Neighborhood, family, and subjective socioeconomic status: How do they relate to adolescent health? Health Psychol 2006; 25(6):704-714.

15. Fratiglioni L, Winblad B, von Strauss E. Prevention of Alzheimer's disease and dementia. Major findings from the Kungsholmen Project. Physiol Behav 2007; 92(12):98-104.

16. Karp A, Kåreholt I, Qiu C, Bellander T, Winblad B, Fratiglioni L. Relation of education and occupation-based socioeconomic status to incident Alzheimer's disease. Am J Epidemiol 2004; 159(2):175-183.

17. Fratiglioni L, Rocca WA. Epidemiology of dementia. In: Boller F, Cappa SF, editors. Handbook of neuropsycholo$g y .2^{\text {nd }}$ ed. Amsterdam: Elsevier; 2001. p. 193- 215.

18. Evans DA, Hebert LE, Beckett LA, Scherr PA, Albert MS, Chown MJ, Taylor JO. Education and other measures of socioeconomic status and risk of incident Alzheimer disease in a defined population of older persons. Arch Neurol 1997; 54(11):1399-1405.

19. Steptoe A, Marmot M. Socioeconomic status and coronary heart disease: A psychobiological perspective. In: Waite LJ, editor. Aging, health and public policy: Demographic and economic perspectives. New York: Population Council; 2004. (Supplement to Vol. 30). p. 133-152.

20. Colhoun HM, Hemingway H, Poulter NR. Socio-economic status and blood pressure: An overview analysis. J Hum Hypertens 1998; 12(2):91-110.

21. Kaplan GA, Keil JE. Socioeconomic factors and cardiovascular disease: A review of the literature. Circulation 1993; 88(4 Pt 1):1973-1998.

22. Duncan GJ, Magnuson KA. Can family socioeconomic resources account for racial and ethnic test score gaps? Future Child 2005; 15(1):35-54.

23. Purcell-Gates V, McIntyre E, Freppon PA. Learning written storybook language in school: A comparison of low - SES children in skills - based and whole language classrooms. American Educational Research Journal 1995; 32(3):659-685.

24. Hochschild JL. Social class in public schools. Journal of Social Issues 2003; 59(4):821-840.

25. Zhang M. Links between school absenteeism and child poverty. Pastoral Care in Education 2003; 21(1):10-17.

26. Harris E. Key strategies to improve schools: How to apply them contextually. Lanham: Scarecrow Press; 2005.

27. Trickett PK, Aber JL, Carlson V, Cicchetti D. Relationship of socioeconomic status to the etiology and developmental sequelae of physical child abuse. Developmental Psychology 1991; 27(1):148-158.

28. Ondersma SJ. Predictors of neglect within low - SES families: The importance of substance abuse. Am J Orthopsychiatry 2002; 72(3):383-391.

29. Melki IS, Beydoun HA, Khogali M, Tamim H, Yunis KA. Household crowding index: A correlate of socioeconomic status and interpregnancy spacing in an urban setting. J Epidemiol Community Health 2004; 58(6):476-480.
30. United Nations Children's Fund (UNICEF). 2013. [acessado 2013 jun 12]. Disponível em: http://www. unicef.org/sowc2013/files/SWCR2013_ENG_Lo_ res_24_Apr_2013.pdf

31. Matos M, Gaspar T, Simões C. Health-Related Quality of Life in Portuguese Children and Adolescents. Psicologia: Reflexão e Crítica 2012; 25(2):230-237.

32. Grotberg EH. Resilience programs for children in disaster. Ambulatory Child Health 2001; 7(2):75-83.

33. Pinto-Coelho J, Gaspar T, Albergaria F. Resilience Scale for Children and Adolescent: Portuguese adaptation and validation. Children and Youth Services Review (submitted).

34. Scheier MF, Carver CS, Bridges MW. Distinguishing optimism from neuroticism (and trait anxiety, self-mastery, and self-esteem): A reevaluation of the Life Orientation Test. J Pers Soc Psychol 1994; 67(6):1063-1078.

35. Kelley RM, Denny G, Young M. Abbreviated Hare self-esteem scale: Internal consistency and factor analysis. American Journal of Health Studies1997; 13(4):180186.

36. Gaspar T, Tomé G, Albergaria F, Freire T. Self-esteem Scale for Children and Adolescent: Portuguese adaptation and validation. (in preparation).

37. Ribeiro J. Escala de Satisfação com o Suporte Social (ESSS). Análise Psicológica 1999; 3(17):574-588.

38. Gaspar T, Ribeiro J, Matos MG, Leal I, Ferreira A. Psychometric Properties of a Brief Version of the Escala de Satisfação com o Suporte Social for Children and Adolescents. The Spanish Journal of Psychology 2009; 12(1):360-372.

39. Dawson M, Pooley JA. Resilience: The role of optimism, perceived parental autonomy support and perceived social support in first year university students. Journal of Education and Training Studies 2013; 1(2):38-49.

40. Harding L. Children's Quality of Life Assessments: a review of genetic and health related quality of life measures completed by children and adolescents. Clinical Psychology and Psychotherapy 2001; 8:79-96.

41. Utsey SO, Hook JN, Belvet B, Fischer N. Influence of cultural orientation, ego resilience, and optimism on the subjective well-being of African Americans. Journal of Positive Psychology 2008; 3(3):202-210.

42. Marujo HA, Neto LM. Álbuns de Família. Manual de Possibilidades sobre o futuro. São Miguel: Instituto de Ação Social; 2007.

43. Bronfenbrenner U. Making human beings human: Bioecological perspectives on human development. Thousand Oaks: Sage; 2005.

44. Bronfenbrenner U. Human development, bioecological theory of. In: Smelser NJ, Baltes PB, editors. International encyclopaedia of the social and behavioural sciences. Oxford: Elsevier; 2001. p. 6963-6970.

45. Eurochild. Relatório anual. 2012 [acessado 2013 jun 7]. Disponível em: http://www.eurochild.org/fileadmin/Communications/10_Annual\%20Reports/Eurochild_Annual_Report_2011.pdf

Artigo apresentado em 06/05/2015

Aprovado em 23/10/2015

Versão final apresentada em 25/10/2015 